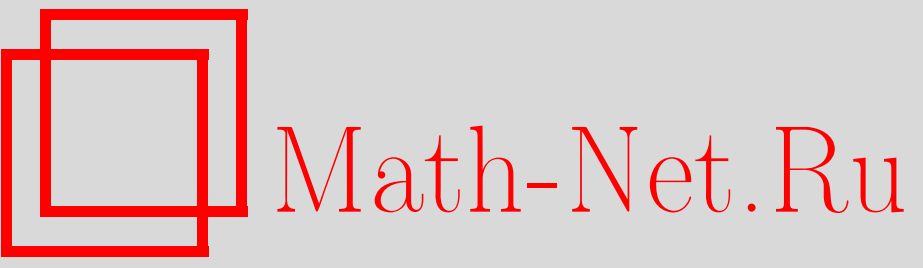

Общероссийский математический портал

М. А. Чеботарь, О решениях функциональных тождеств, Матем. заметки, 2003, том 74, выпуск 6, 949-953

DOI: https://doi.org/10.4213/mzm604

Использование Общероссийского математического портала Math-Net.Ru подразумевает, что вы прочитали и согласны с пользовательским соглашением http://www.mathnet.ru/rus/agreement

Параметры загрузки:

IP : 54.164 .48 .24

26 апреля 2023 г., 16:55:38 


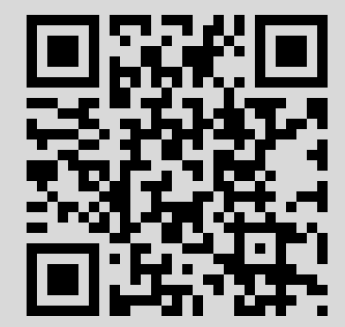




\section{О РЕШЕНИЯХ ФУНКЦИОНАЛЬНЫХ ТОЖДЕСТВ}

\section{М.А. Чеботарь}

Функциональные тождества степени 2 и обобщенные функциональные тождества степени 2 были исследованы в работах Брешара [1], [2]. Понятие функционального тождества произвольной степени было введено Бейдаром [3], а понятие обобщенного функционального тождества произвольной степени было введено автором [4].

С основными результатами теории функциональных тождеств, а также их приложениями можно ознакомиться в обзоре Брешара [5].

Мы будем использовать следующие обозначения (см. детали в [6], [7]):

$R$ - первичное кольцо,

$Q_{l}(R), Q_{r}(R)$ и $Q_{s}(R)$ - соответственно левое, правое и симметрическое мартиндейловские кольца частных для кольца $R$,

$Q_{m l}(R)$ и $Q_{m r}(R)$ - соответственно левое и правое максимальные кольца частных для кольца $R$,

$C$ - расширенный центроид Мартиндейла кольца $R$,

$R C$ - центральное замыкание кольца $R$.

Пусть $Q$ - некоторое кольцо и $r$ - натуральное число. Для отображения $F: R^{r-1} \rightarrow Q$ и $1 \leqslant$ $i \leqslant r$ определим отображение $F^{i}: R^{r} \rightarrow Q, 1 \leqslant i \leqslant r$, по правилу

$$
F^{i}\left(x_{1}, \ldots, x_{r}\right)=F\left(x_{1}, \ldots, x_{i-1}, x_{i+1}, \ldots, x_{r}\right) \quad \text { для всех } x_{1}, \ldots, x_{r} \in R .
$$

Аналогично, для отображения $p: R^{r-2} \rightarrow Q$ и $1 \leqslant i<j \leqslant r$ мы определим отображение $p^{i j}=$ $p^{j i}: R \rightarrow Q$ по правилу

$$
p^{i j}\left(x_{1}, \ldots, x_{r}\right)=p\left(x_{1}, \ldots, x_{i-1}, x_{i+1}, \ldots, x_{j-1}, x_{j+1}, \ldots, x_{r}\right) .
$$

Для удобства подразумевается, что отображение, определенное на $R^{0}$, является константой из $Q$ и отображение, определенное на $R^{-1}$, является нулевым. Мы будем также писать

$$
\begin{aligned}
F^{i} & \text { вместо } F^{i}\left(x_{1}, \ldots, x_{r}\right), \\
p^{i j} & \text { вместо } p^{i j}\left(x_{1}, \ldots, x_{r}\right), \\
F^{i}\left(x_{k} t\right) & \text { вместо } F^{i}\left(x_{1}, \ldots, x_{k-1}, x_{k} t, x_{k+1}, \ldots, x_{r}\right), \quad \text { где } 1 \leqslant k \leqslant r, \quad k \neq i,
\end{aligned}
$$

(здесь $t \in Q-$ это такой элемент, что $R t \subseteq R$ ).

Основными результатами этой работы являются следующие две теоремы.

(C) M. А. Чевотарь 
Теорема 1. Пусть $m, r, n_{j}, j=1, \ldots, r$, - натуральные числа. Далее, пусть $R-$ первичное кольчо, не удовлетворяющее нетривиальным обобщенным полиномиальным тождествам, $T \in\{R, R C\}$, и пусть $F: R^{r} \rightarrow T-$ такое отображсение, что

$$
F\left(x_{1}, \ldots, x_{r}\right)=\sum_{j=1}^{r} \sum_{i=1}^{n_{j}} p_{j i}^{j}\left(x_{1}, \ldots, x_{r}\right) x_{i} a_{i}^{j}+\sum_{k=1}^{m} \lambda_{k}\left(x_{1}, \ldots, x_{r}\right) b_{k},
$$

для всех $x_{1}, \ldots, x_{r} \in R$, әде

$$
\left\{a_{1}^{j}, \ldots, a_{n_{j}}^{j}\right\}, \quad j=1, \ldots, r, \quad\left\{b_{1}, \ldots, b_{m}\right\}
$$

являются подмнохествами $R$, состоящими из әлементов линейно независимых над $C$,

$$
\begin{aligned}
p_{j i}: & R^{r-1} \rightarrow Q_{m r}(R), & j & =1, \ldots, r, \quad i=1, \ldots, n_{j}, \\
\lambda_{k}: & R^{r} \rightarrow C, & k & =1, \ldots, m,
\end{aligned}
$$

- некоторые отображения. Тогда

$$
p_{j i}: R^{r-1} \rightarrow Q_{r}(T), \quad j=1, \ldots, r, \quad i=1, \ldots, n_{j} .
$$

Теорема 2. Пусть $r$ - натуральное число. Далее, пусть $R-$ первичное кольио, $T \in$ $\{R, R C\}$, и пусть $F: R^{r} \rightarrow T-$ такое отобрахсене, что

$$
F\left(x_{1}, \ldots, x_{r}\right)=\sum_{i=1}^{r} p_{i}^{i}\left(x_{1}, \ldots, x_{r}\right) x_{i}+\lambda\left(x_{1}, \ldots, x_{r}\right)
$$

для всех $x_{1}, \ldots, x_{r} \in R$, əде

$$
p_{i}: R^{r-1} \rightarrow Q_{m r}(R), \quad i=1, \ldots, r, \quad \lambda: R^{r} \rightarrow C
$$

- некоторые отображсния. Тогда либо

$$
p_{i}: R^{r-1} \rightarrow Q_{r}(T), \quad i=1, \ldots, r
$$

либо каждый әлемент кольча $R$ является алгебраическим над $C$ степени $\leqslant r$.

Нам потребуется следующий вспомогательный результат.

Лемма 3. Пусть $n$ - натуральное число, $R$ - первичное кольцо, $T \in\{R, R C\}$ и $F: R \rightarrow$ T- отображение, заданное по правилу

$$
F(x)=\sum_{i=1}^{n} a_{i} x b_{i} \quad \text { для всех } \quad x \in R
$$

əде $\left\{a_{1}, \ldots, a_{n}\right\}$ u $\left\{b_{1}, \ldots, b_{n}\right\}$ являются подмножествами $Q_{m r}(R)$, состоящими из әлементов, линейно независимых над $C$. Тогда $a_{i} \in Q_{r}(T)$ для всех $i=1, \ldots, n$ и $b_{i} \in Q_{l}(T)$ для всех $i=1, \ldots, n$.

ДокАЗАТЕльство. Фиксируем произвольное $j, 1 \leqslant j \leqslant n$. По [6, теорема 2.3.3] существуют $\alpha_{k}, \beta_{k} \in R, k=1, \ldots, m$, такие, что

$$
b=\sum_{k=1}^{m} \alpha_{k} b_{j} \beta_{k} \neq 0, \quad \sum_{k=1}^{m} \alpha_{k} b_{i} \beta_{k}=0 \quad \text { для всех } i \neq j .
$$

Из (3) следует, что

$$
\sum_{k=1}^{m} F\left(x \alpha_{k}\right) \beta_{k}=a_{j} x b \in T
$$

для всех $x \in R$. Так как $b \neq 0$, существует такой элемент $a \in R$, что $0 \neq b a \in R$. Таким образом, $a_{j} x b a y \in T$ для всех $x \in R, y \in R$ и $a_{j} \in Q_{r}(T)$ по определению. Аналогично доказывается, что $b_{i} \in Q_{l}(T)$ для всех $i=1, \ldots, n$. 
ДоКАЗАТЕЛЬСтво тЕОРЕмЫ 1. Докажем, что $p_{11}: R^{r-1} \rightarrow Q_{r}(T)$. Для остальных отображений результат будет следовать при соответствующей перестановке индексов в (1).

Индукция по $m$. Пусть $m=0$, т.е. вторая сумма в (1) нулевая.

Индукция по $r$. При $r=1$ все следует из леммы 3 .

Пусть $r>1$. Из (1) мы получаем

$$
\begin{aligned}
F\left(x_{r} a_{n_{r}}^{r} y\right)-F y a_{n_{r}}^{r}= & \sum_{j=1}^{r-1} \sum_{i=1}^{n_{j}}\left[p_{j i}^{j}\left(x_{r} a_{n_{r}}^{r} y\right) x_{i} a_{i}^{j}-p_{j i}^{j} x_{i} a_{i}^{j} y a_{n_{r}}^{r}\right] \\
& +\sum_{i=1}^{n_{r}-1} p_{r i}^{r} x_{r}\left(a_{n_{r}}^{r} y a_{i}^{r}-a_{i}^{r} y a_{n_{r}}^{r}\right) \in T
\end{aligned}
$$

для всех $x_{1}, \ldots, x_{r}, y \in R$.

По [6, лемма 6.1.8] существует такой элемент $y \in R$, что

$$
\begin{aligned}
& \left\{a_{1}^{j}, \ldots, a_{n_{j}}^{j}, a_{1}^{j} y a_{n_{r}}^{r}, \ldots, a_{n_{j}}^{j} y a_{n_{r}}^{r}\right\}, \quad j=1, \ldots, r-1, \\
& \left\{\left(a_{n_{r}}^{r} y a_{1}^{r}-a_{1}^{r} y a_{n_{r}}^{r}\right), \ldots,\left(a_{n_{r}}^{r} y a_{\left(n_{r}-1\right)}^{r}-a_{\left(n_{r}-1\right)}^{r} y a_{n_{r}}^{r}\right)\right\}
\end{aligned}
$$

являются подмножествами $R$, состоящими из элементов, линейно независимьх над $C$. Индукция по $n_{r}$, примененная к (4), завершает доказательство этого случая.

Пусть $m>0$. Из (1) мы получаем

$$
\begin{aligned}
F y b_{m} y-b_{m} y F y= & \sum_{j=1}^{r} \sum_{i=1}^{n_{j}}\left[p_{j i}^{j} x_{i} a_{i}^{j} y b_{m} y-b_{m} y p_{j i}^{j} x_{i} a_{i}^{j} y\right] \\
& +\sum_{i=1}^{m-1} \lambda_{i}\left(b_{i} y b_{m} y-b_{m} y b_{i} y\right) \in T
\end{aligned}
$$

для всех $x_{1}, \ldots, x_{r}, y \in R$. По [6, лемма 6.1.8] существует такой элемент $y \in R$, что

$$
\begin{gathered}
\left\{a_{1}^{j} y b_{m} y, \ldots, a_{n_{j}}^{j} y b_{m} y, a_{1}^{j} y, \ldots, a_{n_{j}}^{j} y\right\}, \quad j=1, \ldots, r, \\
\left\{\left(b_{1} y b_{m} y-b_{m} y b_{1} y\right), \ldots,\left(b_{m-1} y b_{m} y-b_{m} y b_{m-1} y\right)\right.
\end{gathered}
$$

являются подмножествами $R$, состоящими из элементов, линейно независимых над $C$.

Применяя индуктивное предположение по $m$ к (5) получаем, что $p_{11}: R^{r-1} \rightarrow Q_{r}(T)$. Доказательство завершено.

Аналогичным образом доказывается

Теорема 4. Пусть $m, r, n_{j}, j=1, \ldots, r$, - натуральные числа. Далее, пусть $R$ первичное кольчо, не удовлетворяющее нетривиальным обобщенным полиномиальным тохсдествам, $T \in\{R, R C\}$, и пусть $F: R^{r} \rightarrow T-$ такое отобрахение, что

$$
F\left(x_{1}, \ldots, x_{r}\right)=\sum_{j=1}^{r} \sum_{i=1}^{n_{j}} a_{i}^{j} x_{i} p_{j i}^{j}\left(x_{1}, \ldots, x_{r}\right)+\sum_{k=1}^{m} \lambda_{k}\left(x_{1}, \ldots, x_{r}\right) b_{k}
$$

для всех $x_{1}, \ldots, x_{r} \in R$, əде

$$
\left\{a_{1}^{j}, \ldots, a_{n_{j}}^{j}\right\}, \quad j=1, \ldots, r, \quad\left\{b_{1}, \ldots, b_{m}\right\}
$$

являются подмножествами $R$, состоящими из әлементов, линейно независимых над $C$,

$$
\begin{aligned}
p_{j i}: & R^{r-1} \rightarrow Q_{m r}(R), & j & =1, \ldots, r, \quad i=1, \ldots, n_{j}, \\
\lambda_{k} & : R^{r} \rightarrow C, & k & =1, \ldots, m,
\end{aligned}
$$

- некоторые отображения. Тогда

$$
p_{j i}: R^{r-1} \rightarrow Q_{l}(T), \quad j=1, \ldots, r, \quad i=1, \ldots, n_{j} .
$$

Следствие 5 , являющееся основным результатом работы [8], вытекает из [9], теоремы 1 и теоремы 4 . 
СлЕДСтвиЕ 5. Пусть $r, n_{i}, m_{i}, i=1, \ldots, r,-$ натуральные числа. Далее, пусть $R-$ первичное кольчо, $T \in\{R, R C\}, V$ - конечномерное подпространство линейного пространства $Q_{m r}(R)$ над $C$, и пусть $E_{j i}, F_{l k}: R^{r-1} \rightarrow T$ - отобрахсения такие, что

$$
\sum_{j=1}^{r} \sum_{i=1}^{n_{j}} E_{j i}^{j}\left(x_{1}, \ldots, x_{r}\right) x_{j} a_{i}^{j}+\sum_{l=1}^{r} \sum_{k=1}^{m_{l}} b_{k}^{l} x_{l} F_{l k}^{l}\left(x_{1}, \ldots, x_{r}\right) \in V
$$

для всех $x_{1}, \ldots, x_{r} \in R$, əде

$$
\left\{a_{1}^{j}, \ldots, a_{n_{j}}^{j}\right\}, \quad\left\{b_{1}^{j}, \ldots, b_{m_{j}}^{j}\right\}, \quad j=1, \ldots, r
$$

являются подмножествами $R$, состоящими из әлементов, линейно независимых над $C$. Тогда выполнено одно и только одно из следующих двух утверждений:

(i) $R$ - кольцо, удовлетворяющ,е нетривиальным обобщенным полиномиальным тожсдествам;

(ii) существуют и единственнь отображения $p_{j i l k}: R^{r-2} \rightarrow Q_{s}(T) u \lambda_{i l k}: R^{r-1} \rightarrow$ C такие, что

$$
\begin{aligned}
& E_{j i}^{j}=\sum_{\substack{1 \leqslant l \leqslant r \\
l \neq j}} \sum_{k=1}^{m_{l}} b_{k}^{l} x_{l} p_{j i l k}^{j l}+\sum_{k=1}^{m_{j}} \lambda_{i j k}^{j} b_{k}^{j}, \\
& F_{l k}^{l}=-\sum_{\substack{1 \leqslant j \leqslant r \\
j \neq l}} \sum_{i=1}^{n_{j}} p_{j i l k}^{j l} x_{i} a_{i}^{j}-\sum_{i=1}^{n_{l}} \lambda_{i l k}^{l} a_{i}^{l} ;
\end{aligned}
$$

в частности,

$$
\sum_{j=1}^{r} \sum_{i=1}^{n_{j}} E_{j i}^{j} x_{j} a_{i}^{j}+\sum_{l=1}^{r} \sum_{k=1}^{m_{l}} b_{k}^{l} x_{l} F_{l k}^{l}=0
$$

более того, если все отображения $E_{j i}$ и $F_{l k}$ аддитивны по каждому из аргументов, то то же самое справедливо для отобрахений $p_{j i l k} u \lambda_{i l k}$.

ДоКАЗАТЕЛЬСтво теоремы 2. Докажем, что $p_{1}: R^{r-1} \rightarrow Q_{r}(T)$. Для остальных отображений результат будет следовать при соответствующей перестановке индексов в (2).

Пусть $t \in R$ - элемент степени $>r$ над $C$.

Индукция по $r$. Пусть $r=1$. Из (2) получаем, что

$$
[F, t]=F t-t F=p_{1}^{1} x_{1} t-t p_{1}^{1} x_{1} \in R,
$$

и требуемый результат следует из леммы 3 .

Пусть $r>1$. Из (2) получаем, что

$$
G_{1}=[F, t]=F t-t F=\sum_{i=1}^{r} p_{i}^{i} x_{i} t-\sum_{i=1}^{r} t p_{i}^{i} x_{i}
$$

для всех $x_{1}, \ldots, x_{r} \in R$.

Положим $G_{k}=G_{k} t-G_{k}\left(x_{k} t\right), k=2, \ldots, r$. Имеем

$$
G_{r}=\sum_{i=0}^{r}(-1)^{i} t^{i} p_{1}^{1} x_{1} t^{r-i} \in R
$$

для всех $x_{1}, \ldots, x_{r} \in R$.

По лемме $3 p_{1}: R^{r-1} \rightarrow Q_{r}(T)$. Доказательство завершено.

Аналогично доказьвается 
Теорема 6. Пусть $r$ - натуральное число. Далее, пусть $R-$ первичное кольчо, $T \in$ $\{R, R C\}$, и пусть $F: R^{r} \rightarrow T-$ такое отображсние, что

$$
F\left(x_{1}, \ldots, x_{r}\right)=\sum_{i=1}^{r} x_{i} p_{i}^{i}\left(x_{1}, \ldots, x_{r}\right)+\lambda\left(x_{1}, \ldots, x_{r}\right)
$$

для всех $x_{1}, \ldots, x_{r} \in R$, где $p_{i}: R^{r-1} \rightarrow Q_{m r}(R), i=1, \ldots, r, u \lambda: R^{r} \rightarrow C$ - некоторые отображения. Тогда либо $p_{i}: R^{r-1} \rightarrow Q_{l}(T), i=1, \ldots, r$, либо каждьй элемент кольча $R$ является алгебраическим над $C$ степени $\leqslant r$.

Следствие 7 вытекает из [3], теоремы 2 и теоремы 6.

СледСтвиЕ 7. Пусть $R$ - первичное кольцо, $T \in\{R, R C\}$ и $r$ - натуральное число. Пусть $E_{i}, F_{i}: R^{r-1} \rightarrow T, i=1, \ldots, r,-$ произвольные отобрахения. Полохим

$$
\pi\left(x_{1}, \ldots, x_{r}\right)=\sum_{i=1}^{r} E_{i}^{i}\left(x_{1}, \ldots, x_{r}\right) x_{i}+\sum_{j=1}^{r} x_{j} F_{j}^{j}\left(x_{1}, \ldots, x_{r}\right) .
$$

(a) $\operatorname{Ecли~} \pi\left(x_{1}, \ldots, x_{r}\right) \in C$ для всех $x_{1}, \ldots, x_{r} \in R$, тогда выполнено одно и только одно из следуюших двух утверхдений:

(i) каждый әлемент кольца $R$ является алъебрачческим над $C$ степени $\leqslant r$;

(ii) $\pi\left(x_{1}, \ldots, x_{r}\right)=0$ для всех $x_{1}, \ldots, x_{r} \in R$.

(b) Если $\pi\left(x_{1}, \ldots, x_{r}\right)=0$ для всех $x_{1}, \ldots, x_{r} \in R$, тогда выполнено одно и только одно из следующ,их двух утверждений:

(iii) каждый әлемент кольча $R$ является алгебраическим над $C$ степени $\leqslant r-1$;

(iv) существуют и единственны отображсения $p_{i j}: R^{r-2} \rightarrow Q_{s}(T) u \lambda_{i}: R \rightarrow C$ такuе, что

$$
E_{i}^{i}=\sum_{\substack{1 \leqslant j \leqslant r \\ j \neq i}} x_{j} p_{i j}^{i j}+\lambda_{i}^{i}, \quad F_{j}^{j}=\sum_{\substack{1 \leqslant i \leqslant r \\ i \neq j}}-p_{i j}^{i j} x_{i}-\lambda_{j}^{j}
$$

более того, если все отображения $E_{i}$ и $F_{j}$ аддитивны по каждому из аргументов, то то же самое справедливо для отображений $p_{i j} и \lambda_{i}$.

\section{СПИСОК ЦИТИРОВАННОЙ ЛИТЕРАТУРЫ}

1. Brešar M. // J. Algebra. 1995. V. 172. P. 764-786. 2. Brešar M. // J. Algebra. 1995. V. 172. P. 690-720. 3. Beidar K. I. // Comm. Algebra. 1998. V. 26. P. 1819-1850. 4. Chebotar M. A. // J. Algebra. 1998. V. 202. P. 655-670. 5. Brešar M. // Contemporary Math. 2000. V. 259. P. 93-109. 6. Beidar K. I., Martindale W.S., Mikhalev A. V. Rings with Generalized Identities. New York: Marcel Dekker, Inc., 1996. 7. Martindale W. S. // J. Algebra. 1969. V. 12. P. 576-584. 8. Yu W. // Comm. Algebra. 2002. V. 30. P. 2897-2913. 9. Beidar K. I., Brešar M., Chebotar M. A. // J. Algebra. 1999. V. 215. P. 644-665. 